\title{
Optimization of soft $x$-ray amplifiers by controlling plasma hydrodynamics
}

\author{
E.Oliva $^{* \dagger}$, Ph. Zeitoun**, P. Velarde*, M. Fajardo ${ }^{\star}$, K. Cassou ${ }^{\S}$, D. Ros \\ and S. Sebban** \\ *Instituto de Fusión Nuclear, Universidad Politécnica de Madrid, Madrid, Spain \\ ${ }^{\dagger}$ Laboratoire d'Optique Apliquée, ENSTA ParisTech,École Polytechnique, CNRS UMR \\ 7639,91761 Palaiseau Cedex, France \\ ** Laboratoire d'Optique Apliquée, ENSTA ParisTech,Ecole Polytechnique, CNRS UMR \\ 7639,91761 Palaiseau Cedex, France \\ ${ }^{\ddagger}$ Centro de Física dos Plasmas, Instituto Superior Técnico, Lisbon, Portugal; \\ ${ }^{\S}$ Laboratoire de Physique des Gaz et des Plasmas, CNRS, Université Paris Sud XI,91405 Orsay \\ Cedex, France \\ 'Laboratoire d'Interaction du Rayonnement X avec la Matière, CNRS, Université Paris SudXI, \\ 91405 Orsay,France
}

\begin{abstract}
Several coherent soft x-ray lasers are available for applications nowadays. Among them, plasma-based soft x-ray lasers promise to generate high-energy, highly coherent, short pulse beam. Solid target based amplifiers, due to the fact that his density is higher, should store a higher amount of energy. However, to-date output energy from seeded solid amplifiers remains as low as $60 \mathrm{~nJ}$. We demonstrated that the extraction of the energy stored in the plasma is enhanced by carefully tailoring the plasma shape, to inhibit deleterious hydrodynamical effects. With $1 \mathrm{~mm}$ wide plasma, energy as high as $22 \mu J$ in sub-ps pulse is achievable. Not only the energy extracted is higher in these tailored plasmas but also gain and pumping efficiency are increased by nearly a factor of ten as compared to the narrowest plasma amplifiers studied previously and here.
\end{abstract}

Keywords: Seeded Soft X-Ray Lasers, plasma amplifiers, Transient Collisional Excitation, Adaptive Mesh Refinement, CFD

PACS: $42.55 . \mathrm{Vc}$

\section{INTRODUCTION}

Nowadays, interest in high brightness sources of coherent soft X-ray radiation is growing, as there are many exciting applications in various fields from Biology [1] to Physics [2]. FEL, seeded plasma-based soft X-ray lasers or High Harmonics are currently available for them. However, many breakthrough experiments envisaged today are demonstrated only on FEL since they require energy per pulse exceeding $10 \mu J$ to produce ultra-high intensity [3] near or above $10^{16} \mathrm{~W} / \mathrm{cm}^{2}$ or to perform single-shot images [4] of a fast evolving sample. Within the panorama of intense soft $x$-ray sources, plasmabased soft $x$-ray lasers are very attractive since they demonstrated the highest energy per pulse, up to $10 \mathrm{~mJ}$ [5], however from a weakly coherent source, emitting pulses of several hundredths of picoseconds. A promising technique to extract the high energy stored in plasma-based soft x-ray laser while having sources of very good optical quality consists on seeding the plasma amplifier with High Order Harmonics radiation $[6,7,8,9,10,11,12]$. Saturation in an Optical Field Ionization (OFI) amplifier 
has been recently demonstrated [7] obtaining an amplified beam up to $700 \mathrm{~nJ}$. The low density of these amplifiers limits the energy which can be extracted and thus the maximum brightness of the beam. For these reasons, solid amplifiers have to be used. The combination of Transient Collisional Excitation (TCE) [13] with novel techniques as GRazing Incidence Pumping GRIP [14] has allowed to demonstrate saturated amplification in solid targets $[9,10]$, obtaining a beam of $\approx 75 n J$. Although table-top TCE-GRIP schemes are a user-friendly technique, there is still much work to optimize these sources.

Computational modeling is a powerful tool to comprehend the physics of these amplifiers and to optimize the sources. Recently, the influence of transverse spatial laser driver profiles in the gain region has been studied [15], with the demonstration that 2D hydrodynamic effects have a strong impact in the amplification region. A super-Gaussian profile enhances gain values and enlarges the area where population inversion takes place. In this paper we continue the modeling work above mentioned studying the evolution of plasmas created with different transverse spatial laser widths (i. e. the Full Width at Half Maximum of the super-Gaussian), ranging from $20 \mu \mathrm{m}$ to $1 \mathrm{~mm}$.

The simulations have been done with the two-dimensional hydrodynamic code ARWEN [16] with radiation transport, based on Adaptive Mesh Refinement[17, 18] (AMR). The hydrodynamic data obtained with the ARWEN code (electron density and temperature, fluid velocities, etc ...) has been postprocessed using a simple three-level atomic model [15] to obtain several data as gain peak and saturation fluence maps among others.

The layout of the paper will be as follows: in the first section we will present the computational tools (the ARWEN code and the atomic model) used. In next section we will describe the simulations we have done. The last section is dedicated to show the results obtained and explain the physics that drives these processes. Finally, the conclusions obtained from the simulations will be presented.

\section{COMPUTATIONAL TOOLS}

The main program is the ARWEN code [16]. The simple atomic level was originally written for [15] and has been modified for this paper to facilitate the postprocessing of the ARWEN data.

\section{The ARWEN code}

The ARWEN code is a 2D hydrodynamic code with radiation transport, developed at the Instituto de Fusión Nuclear of the Universidad Politécnica de Madrid. Fluid dynamics equations are solved using a high order Godunov method. Radiation transport is treated by a $S_{n}$ multigroup method and flux limited electron thermal conduction is solved by multigrid methods. 2D ray-tracing subroutines to take into account the incident laser refraction in the plasma are available. Simulations can be done in planar and cylindrical geometries.

Tables of Equation of State and Opacities are generated prior to the simulations in order to save computational time. The EOS is obtained from QUEOS [19] and opacities 
are generated with JIMENA [20], also developed at the Instituto de Fusión Nuclear.

The ARWEN code uses the Adaptive Mesh Refinement technique [17, 18]. This technique is very useful in saving computational time while retaining a high accuracy in the areas where it is needed, by patching these zones with finer meshes. The structure of levels and grids is created and controlled by the $\mathrm{C}++$ library Boxlib [21].

\section{The atomic model}

In order to compute several parameters of interest as gain and saturation fluence, the hydrodynamic data obtained from ARWEN (i.e. electron and ion density and temperature) are postprocessed to obtan the data needed. This postprocess is done with a simple three-level atomic model which computes the $2 p_{\frac{1}{2}}^{5} 3 s_{\frac{1}{2}} \mathrm{~J}=1 \rightarrow 2 p_{\frac{1}{2}}^{5} 3 p_{\frac{1}{2}} \mathrm{~J}=0$ Neonlike $F e^{16+}$ transition occuring at $\lambda=25.5 \mathrm{~nm}$. In our model we assume that the fundamental level $(0)$ is much more populated than the other two levels. There is a forbidden transition between the fundamental level and the upper lasing level (2), allowing to create a population inversion between the two lasing levels $(1,2)$ by collisional excitation. The mechanisms taken into account in our model are collisional excitation between the fundamental and the two lasing levels $(0 \rightarrow 2,0 \rightarrow 1)$ and collisional deexcitation and radiative transitions $(2 \rightarrow 1,1 \rightarrow 0)$. With this assumptions, we can compute the population inversion.

The stimulated emission cross section is computed as:

$$
\sigma_{\text {stim }}(v)=\Phi(v) \frac{\lambda^{2}}{8 \pi} A_{21}
$$

where $A_{21}$ is the Einsteint's coefficient and $\Phi(v)$ is the line profile. We assume a Doppler profile, but a new version of the subroutine can take into account collisional broadening. In addition to this, we will only compute the value of gain at the center of the line profile (i. e. $v=0$ for a normalized profile). Now, we can compute the gain coefficient and saturation fluence:

$$
\begin{aligned}
g_{0} & =\left(N_{2}-\frac{g_{2}}{g_{1}} N_{1}\right) \sigma_{\text {stim }} \\
F_{\text {sat }} & =\frac{h v(0)}{\sigma_{\text {stim }}}
\end{aligned}
$$

\section{SIMULATIONS}

We will simulate amplifiers pumped by Transient Collisional Excitation scheme [13]. Our model consist on three laser pulses arriving at 1.5,2 and 2.51 ns from the start of the simulation. The temporal FWHM are respectively $1000 \mathrm{ps}, 100 \mathrm{ps}$ and $0.1 \mathrm{ps}$. The first pulse creates a weakly ionized plasma which enhances the absorption of the second pulse (main pulse) which ionizes the plasma. The last pulse heats the electrons while the ions continue freezed in its state. The kinetic energy of the electrons is thus increased and therefore the pumping rate, allowing to create the population inversion. 
Recently, modeling aiming to optimize the amplification region has been published [15] concluding that 2D effects, driven by the transverse spatial laser profile, have a strong impact on plasma hydrodynamics. Super-Gaussian profile enlarges the gain zone surface and reduces the transverse refraction as energy deposition along the transverse direction ( $\mathrm{X}$ axis) enhances laser coupling to the plasma and reduces energy loss due to lateral plasma cooling. For this reason, all lasers have a super-gaussian $I=I_{0} \exp \left[-y^{2} /\left(2 \sigma^{2}\right)\right]^{n}$ spatial profile, with $n=10$ as suggested in [15]. The FWHM has been varied from $20 \mu \mathrm{m}$ to $1 \mathrm{~mm}$. For the sake of comparison we keep for every plasma width the intensities constant at these values: $1.25 \times 10^{11} \mathrm{~W} / \mathrm{cm}^{2}$ for the $1 \mathrm{~ns}$ pulse, $1.25 \times 10^{12} \mathrm{~W} / \mathrm{cm}^{2}$ and $1.16 \times 10^{15} \mathrm{~W} / \mathrm{cm}^{2}$ for the $100 \mathrm{ps}$ and 0.5 ps pulses respectively. The laser wavelength is $\lambda=800 \mathrm{~nm}$. The target is an iron slab, as the atomic properties and equations of state of iron are well known.

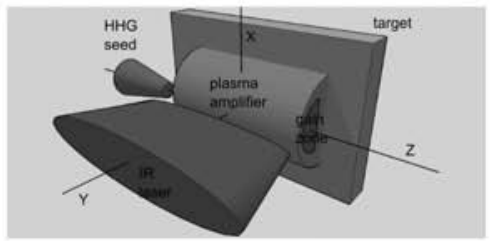

FIGURE 1. Scheme of the simulations and the coordinate system used in the paper

\section{COMPUTATIONAL RESULTS}

As explained in section, simulations with different linewidths ranging from $20 \mu \mathrm{m}$ to $1 \mathrm{~mm}$ have been done. The hydrodynamic data obtained is postprocessed to obtain gain and saturation fluence. It is worth noting that our values of gain and saturation fluence where gain takes place are in very good accord with the experimental values found in Wang et al experiments $[9,8]$.

TABLE 1. Gain peak and saturation fluence.

\begin{tabular}{ccccccccc} 
Width $(\mu \mathrm{m})$ & 20 & 30 & 50 & 75 & 100 & 150 & 200 & 1000 \\
\hline Gain $\left(\mathrm{cm}^{-1}\right)$ & 13 & 60 & 80 & 110 & 124 & 126 & 126 & 126 \\
$F_{\text {sat }}\left(\mathrm{mJ} / \mathrm{cm}^{2}\right)$ & 2.22 & 2.28 & 2.40 & 2.50 & 2.50 & 2.56 & 2.63 & 2.61
\end{tabular}

In table 1 are shown the maximum values of gain achieved in the simulations done. A correlation between gain and linewidth is found. For $20 \mu \mathrm{m}$ width we only obtain gain of $13 \mathrm{~cm}^{-1}$. Then, gain increases rapidly with the linewidth until it achieves it maximum value of $126 \mathrm{~cm}^{-1}$ for $150 \mu \mathrm{m}$ width. Further increments of linewidth do not augment the maximum gain value, as we keep constant the intensity of the lasers.

A much more striking correlation is observed on the 2D false color maps of gain: the extension of the gain zone in Y direction is enlarged with the linewidth. In Fig. 2 are depicted the three most relevant cases: $30(\mu \mathrm{m}), 150(\mu \mathrm{m})$ and $1 \mathrm{~mm}$ widths.

Both effects are clearly seen in Fig. 2. The $30 \mu \mathrm{m}$ case presents much more lower gain and vertical (Y) extension of the gain zone, whereas the other two cases have the same values of gain and vertical extension at the center of the plasma $(\mathrm{X}=0 \mu \mathrm{m})$. 

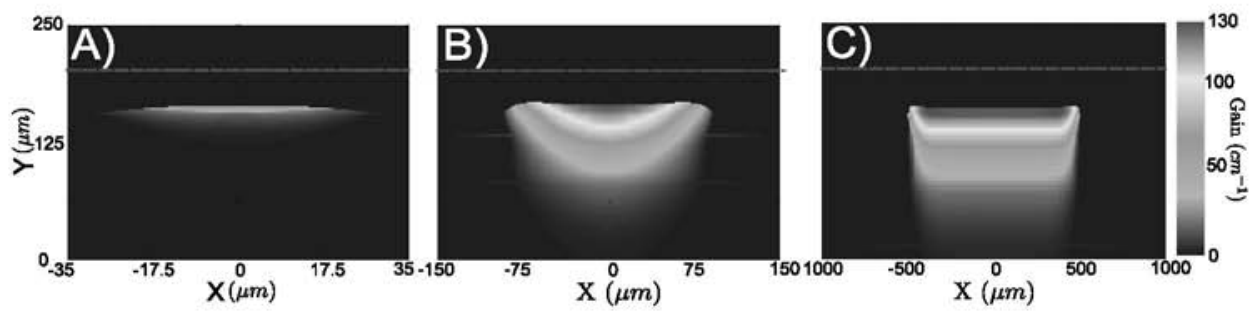

FIGURE 2. Gain maps in false colors for $30 \mu \mathrm{m}$ (left), $150 \mu \mathrm{m}$ (centre) and $1 \mathrm{~mm}$ (right) linewidth. The three figures have the same vertical scale for convenience of comparison. The red line indicates the initial position of the target. The pump lasers come from the bottom to the top

The third difference shown in the pictures is the key to explain these results. In the 30 $\mu m$ case (fig. 2.a) the horizontal extension of gain is much wider than the focal linewidth, indeed it is almost one and a half wider (approximately $40 \mu \mathrm{m}$ ). This effect is observed with less intensity in the $150 \mu \mathrm{m}$ case (fig. 2.b) whereas a clear curvature on the gain zone appears. Finally, in the $1 \mathrm{~mm}$ case (fig. 2.c) the width of the gain zone is the same as the focal linewidth, having the gain zone a rectangular shape. The conclusion is that not only the expansion in the $\mathrm{Y}$ axis plays a role in the gain but also the lateral expansion in the $\mathrm{X}$ axis, widening the gain zone in the cases where these $2 \mathrm{D}$ effects are strong (i.e. in narrower plasmas). For wider plasmas these $2 \mathrm{D}$ effects do not affect the majority of the gain zone, being its behaviour 1D.

The lateral expansion (a hydrodynamic process) and the gain creation (an atomic process) are linked by electron density. Electron density is a hydrodynaimic parameter, influenced by the evolution of the plasma, and drives collisional excitation which is the main process involved in the creation of the population inversion. In Fig. 3, vertical profiles at $X=0 \mu \mathrm{m}$ of electron density are plotted. As shown in Fig. 3, electron density

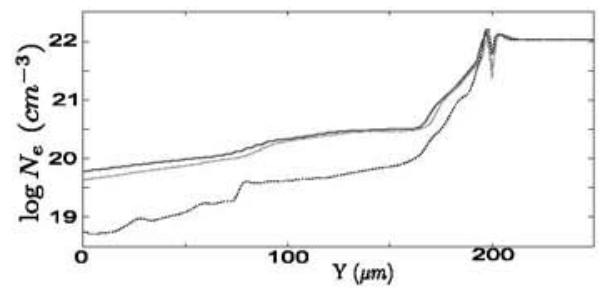

FIGURE 3. Electron density profile in Y direction at the center of the plasma $(X=0 \mu \mathrm{m})$ for $30 \mu \mathrm{m}$ (blue), $150 \mu \mathrm{m}$ (green) and $1 \mathrm{~mm}$ (red) widths. The plasma expands from right to left.

is much smaller for the $30 \mu \mathrm{m}$ plasma, having the same profile at the center of the plasma $(X=0 \mu m)$ for the two other cases.

In Fig. 4, 2D maps of electron temperature in false colors and electron density isocontours are depicted.We observe in Fig. 4.a that, not only is the electron density lower in the narrower case, but also the temperature. The absorption of the short pulse (which must heat electrons to create gain) varies with $n_{e}^{3 / 2}$. Then, the last short pulse is not well absorbed in the narrowest plasma (as electron density has low values) 
and electron temperature is lower. Gain cannot take place except in the small zone of relative high density $\left(N_{e} \approx 10^{20} \mathrm{~cm}^{-3}\right)$ and temperature. Then, the extension in $\mathrm{Y}$ direction shortens for the $30 \mu \mathrm{m}$ case. In addition to this, temperature and density are very homogeneous along the $\mathrm{X}$ direction. In Fig. 4.b the plasma is no more homogeneous. Electron density isocontours present some curvature, and are parallel to the $\mathrm{X}$ axis only at the very center of the plasma $(\mathrm{X}=0)$. This explains the curved form of the gain region shown in Fig. 2.b. A strong lateral expansion is taking place and the plasma is fully $2 \mathrm{D}$. Electron temperature is also inhomogeneous as the thermal conduction is greater in $\mathrm{Y}$ direction than in $\mathrm{X}$ direction. Finally, in Fig. 4.c, a large region, which has a 1D behavior
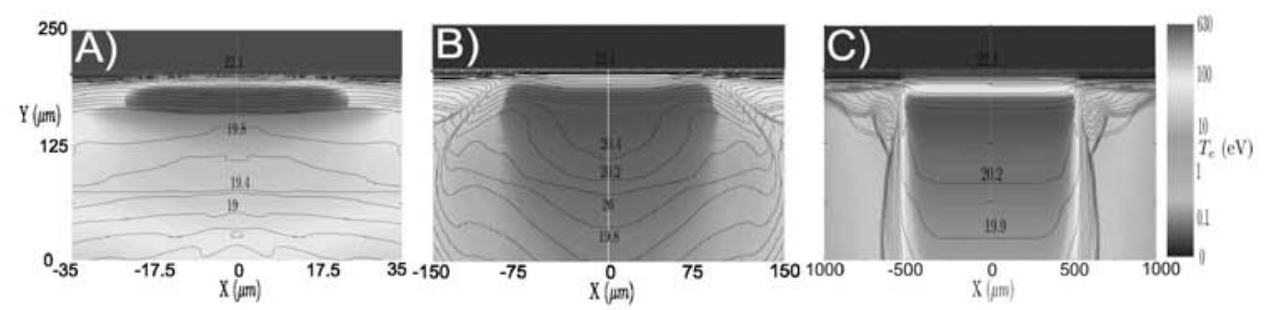

FIGURE 4. Electron temperature maps in false colors and electron density isocontours for $30 \mu \mathrm{m}$ (left), $150 \mu \mathrm{m}$ (centre) and $1 \mathrm{~mm}$ (right) linewidth. The red line indicates the initial position of the target. The pump lasers come from the bottom to the top

(contours are parallel to the $\mathrm{X}$ axis) appears in the center of the plasma. This region is confined by strong lateral density gradients. Temperature is also homogeneous inside this region and lateral thermal conduction is inhibited by these density gradients. The gain is thus confined in this region, which explains that the lateral (X direction) profile of the gain zone in Fig. 2.c presents no wings longer than the linewidt. In Fig. 2.a and Fig. 2.b, these wings appeared as there is some lateral thermal conduction in these zones.
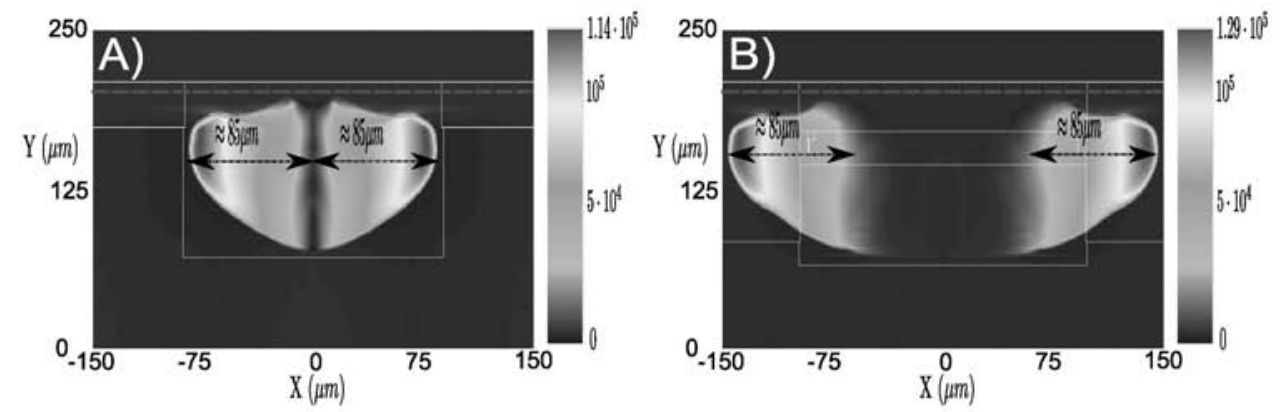

FIGURE 5. Plasma velocities $(\mathrm{m} / \mathrm{s})$ in $\mathrm{X}$ direction at $t=1.5 \mathrm{~ns}$ for $30 \mu \mathrm{m}$ (left) and $150 \mu \mathrm{m}$ (right) widths.

Recapitulating, we have observed that the narrowest plasmas ( $30 \mu \mathrm{m}$ in this case) have low values of electron density and temperature, and present a strong lateral expansion. The wider plasmas $(150 \mu \mathrm{m}$ and $1 \mathrm{~mm})$ present at the center of the plasma the same 
electron density, temperature and gain profile, but several differences far from the center: while the $150 \mu \mathrm{m}$ case presents curved isocontours of density, which denote a lateral expansion of the plasma, the $1 \mathrm{~mm}$ case has a wide $1 \mathrm{D}$ zone. These observations indicate that the lateral expansion of the plasma at its center plays a fundamental role. In Fig. 5 the plasma horizontal velocities at $t=1.5 n$ s (i.e. the moment when the peak of the prepulse arrives) for the $30 \mu \mathrm{m}$ (left) and $150 \mu \mathrm{m}$ (right) widths. It is clearly seen in Fig. 5.a that the fluid around the center of the plasma starts to expand laterally (the velocity around the center is different from zero). This lateral expansion is lowering the electron density from the moment it reaches the center of the plasma until gain is produced $(\approx 1 n s$ in this case), which explains the low density of the narrower plasmas and the homogeneity we found.

On the other hand, for the $150 \mu \mathrm{m}$ case at $t=1.5 \mathrm{~ns}$ (Fig. 5.b), the fluid at the center of the plasma is only expanding vertically (i.e. horizontal velocity is zero). The central region has a $1 \mathrm{D}$ behavior. This is as the size of the region expanding horizontally is the same for all cases at the same time until it reaches the center of the plasma. This size (at $t=1.5 \mathrm{~ns}$ is about $85 \mu \mathrm{m}$ ) is negligible for wider plasmas but not for the narrower.

Now we can explain the effects observed in Fig. 2 and Fig. 4. The prepulse accelerates the plasma in all three cases. The plasma starts to expand laterally independently of the plasma linewidth. At $t=1.5 \mathrm{~ns}$ the lateral expansion arrives to the center of the $30 \mu \mathrm{m}$ plasma. Its behavior will be no more similar to the other two cases, as the density at the center starts to be lowered by this expansion. When the pumping laser pulse arrives, density has low values and gain can only be created in a small region near the target. On the other hand, the $150 \mu \mathrm{m}$ and the $1 \mathrm{~mm}$ widths evolve similarly at the center of the plasma, as the horizontal acceleration does not arrive there. Nevertheless, the region expanding laterally in the $150 \mu \mathrm{m}$ case is not negligible compared with the plasma width, and some curvature in gain appears. The $1 \mathrm{~mm}$ case present a rectangular shaped 1D region expanding only vertically.

We have made rough estimations of the energy that can be extracted and of the efficiency of each amplifier in order to compare the schemes. In the $30 \mu \mathrm{m}$ case, the gain zone size is about $36 \mu \mathrm{m} \times 8 \mu \mathrm{m}$ along $\mathrm{X}$ and $\mathrm{Y}$ directions respectively. Our atomic model gives a saturation fluence of about $F_{\text {sat }}=2.28 \mathrm{~mJ} / \mathrm{cm}^{2}$. As the gain zone has a surface of about $2.9 \times 10^{-6} \mathrm{~cm}^{2}$ we can estimate the saturation energy as $E_{\text {sat }} \approx 6.6 \mathrm{~nJ}$. In reference [7] the measured total output energy was 38 times higher than the saturation energy; in this paper we will use a more conservative value for the output energy equal to ten times the saturation energy. Then, the expected final energy of the beam is about $E \approx 66 n J$, in very good agreement with the maximum energy measured in [8], which is 50-60 nJ. The saturation fluence for the $150 \mu \mathrm{m}$ and $1 \mathrm{~mm}$ plasmas are $2.56 \mathrm{~mJ} / \mathrm{cm}^{2}$ and $2.61 \mathrm{~mJ} / \mathrm{cm}^{2}$ respectively. The areas of the gain region are respectively $1.2 \times 10^{-4} \mathrm{~cm}^{2}$ and $8.6 \times 10^{-4} \mathrm{~cm}^{2}$ leading to output energies (with the same assumptions as in the former case) of $3.1 \mu \mathrm{J}$ and $22.4 \mu \mathrm{J}$. The amount of energy that can be extracted is comparable to the energy achieved on FLASH FEL which is to date the most intense coherent soft X-ray laser source.

To evaluate the efficiency of the plasma, we computed the energy needed to amplify $10^{5}$ times the line center of the seeded HHG. For $20 \mu \mathrm{m}$ width, the length for achieving this amplification is nearly $9 \mathrm{~mm}$ while it drops to $2 \mathrm{~mm}$ for the $30 \mu \mathrm{m}$ width, with the consequence of a huge decrease on pumping energy. It increases gradually again for 
larger plasmas due to the higher amount of matter to heat up. Thus the net pumping energy is $2.5 \mathrm{~J}$ for a $20 \mu \mathrm{m}$ plasma and $1.9 \mathrm{~J}$ and $131 \mathrm{~J}$ for $150 \mu \mathrm{m}$ and $1 \mathrm{~mm}$ plasmas respectively. The pumping efficiency raised strikingly from $4 \mathrm{~nJ} / \mathrm{J}$ for the narrower plasma up to $1.59 \mu \mathrm{J} / \mathrm{J}$ to $1.71 \mu \mathrm{J} / \mathrm{J}$ for $150 \mu \mathrm{m}$ and $1 \mathrm{~mm}$ plasmas.

TABLE 2. Energy of the pumping lasers, extracted energy, gain peak and efficiency

\begin{tabular}{ccccc} 
Width $(\mu m)$ & $E_{\text {input }}(\mathrm{J})$ & $E_{\text {out }}(\mu J)$ & Gain $\left(\mathrm{cm}^{-1}\right)$ & Efficiency $(\mathrm{nJ} / \mathrm{J})$ \\
\hline 20 & 2.5 & 0.01 & 13 & 4 \\
30 & 0.8 & 0.06 & 60 & 80 \\
50 & 1.0 & 0.6 & 80 & 610 \\
75 & 1.1 & 1.1 & 110 & 980 \\
100 & 1.3 & 1.5 & 124 & 1130 \\
150 & 1.9 & 3.1 & 126 & 1590 \\
200 & 2.6 & 4.6 & 126 & 1770 \\
1000 & 13.1 & 22 & 126 & 1710
\end{tabular}

\section{CONCLUSIONS}

We have done several 2D hydrodynamic simulations of different TCE plasmas, continuing the work done in earlier studies $[15,22]$. We have used the $2 \mathrm{D}$ hydrodynamic code with radiation transport in AMR, ARWEN [16] and postprocessed the data obtained with a three-level atomic model, to study the $2 p_{\frac{1}{2}}^{5} 3 s_{\frac{1}{2}} \mathrm{~J}=1 \rightarrow 2 p_{\frac{1}{2}}^{5} 3 p_{\frac{1}{2}} \mathrm{~J}=0$ Neonlike $F e^{16+}$ transition.

Our results have been benchmarked with experiments from Wang et al $[9,8]$, obtaining values of gain, saturation fluence and electron density where population inversion takes place in good accord with the experimental values. Our model suggests that the gain zone is very small because of deleterious hydrodynamical effects and thus the output energy is intrinsically limited to values below $100 \mathrm{~nJ}$. Simulations performed with wider plasmas corroborate this assumption. The lateral expansion at early stages of the evolution of narrower plasmas lowers the density at the center of the plasma, inhibiting the mechanism which creates the population inversion (electron collisional excitation) and thus reducing the gain coefficient and the extension of the gain region. For wider plasmas, the lateral expansion cannot reach the central region before gain takes place. Thus, a novel geometry is proposed, increasing the width and decreasing the length of the plasma so as to extract more energy and to dramatically increase the pumping efficiency. With this geometry, energy as high as $22 \mu J$ can be obtained with a $1 \mathrm{~mm}$ width plasma.

\section{ACKNOWLEDGMENTS}

The authors would like to thank the financial support provided by the ELI-PP212105 project and the Spanish Ministerio de Educación y Ciencia within the program ENE2009-09837/FTN. 


\section{REFERENCES}

1. R. E. Burge, M. T. Browne, P. Charalambous, G. E. Slark, and P. J. Smith, Opt. Lett. 18, 661-663 (1993).

2. J. Itatani, J. Levesque, D. Zeidler, H. Niikura, H. Pépin, J. C. Kieffer, P. B. Corkum, and D. M. Villeneuve, Nature 432, 867-871 (2004).

3. H. Wabnitz, A. de Castro, P. Gürtler, T. Laarmann, W. Laasch, J. Schulz, and T. Möller, Phys. Rev. Lett. 94, 023001 (2005).

4. H. N. Chapman, Nature Materials 8, 299-301 (2009).

5. B. Rus, T. Mocek, A. R. Präg, M. Kozlová, G. Jamelot, A. Carillon, D. Ros, D. Joyeux, and D. Phalippou, Phys. Rev. A 66, 063806 (2002).

6. T. Ditmire, M. H. R. Hutchinson, M. H. Key, C. L. S. Lewis, A. MacPhee, I. Mercer, D. Neely, M. D. Perry, R. A. Smith, J. S. Wark, and M. Zepf, Phys. Rev. A 51, R4337 (1995).

7. P. Zeitoun, G. Faivre, S. Sebban, T. Mocek, A. Hallou, M. Fajardo, D. Aubert, P. Balcou, F. Burgy, D. Douillet, S. Kazamias, G. de Lachèze-Muriel, T. Lefrou, S. le Pape, P. Mercère, H. Merdji, A. S. Morlens, J. P. Rousseau, and C. Valentin, Nature 431, 427 (2004).

8. Y. Wang, E. Granados, F. Pedaci, D. Alessi, B. M. Luther, M. Berrill, and J. J. Rocca, Nature Photonics 2 (2008).

9. Y. Wang, E. Granados, M. A. Larotonda, M. Berrill, B. M. Luther, D. Patel, C. S. Menoni, and J. J. Rocca, Phys. Rev. Lett. 97, 123901 (2006).

10. N. Hasegawa, T. Kawachi, A. Sasaki, M. Kishimoto, K. Sukegawa, M. Tanaka, R. Z. Tai, Y. Ochi, M. Nishikino, K. Nagashima, and Y. Kato, Phys. Rev. A 76, 043805 (2007).

11. F. Pedaci, Y. Wang, M. Berrill, B. Luther, E. Granados, and J. J. Rocca, Opt. Lett. 33, 491-493 (2008), URL http://ol . osa .org/abstract. cfm?URI =ol-33-5-491.

12. Y. Wang, M. Berrill, F. Pedaci, M. M. Shakya, S. Gilbertson, Z. Chang, E. Granados, B. M. Luther, M. A. Larotonda, and J. J. Rocca, Physical Review A (Atomic, Molecular, and Optical Physics) 79, 023810 (2009), URL http: // link . aps .org/abstract/PRA/v79/e023810.

13. P. V. Nickles, V. N. Shlyaptsev, M. Kalachnikov, M. Schnürer, I. Will, and W. Sandner, Phys. Rev. Lett. 78, 2748 (1997).

14. R. Keenan, J. Dunn, P. K. Patel, D. F. Price, R. F. Smith, and V. N. Shlyaptsev, Phys. Rev. Lett. 94, 103901 (2005).

15. K. Cassou, P. Zeitoun, P. Velarde, F. Roy, F. Ogando, M. Fajardo, G. Faivre, and D. Ros, Phys. Rev. A 74, 045802 (2006).

16. F. Ogando, and P. Velarde, J. Quant. Spectrosc. Radiat. Transf. 71, 541 (2001).

17. M. J. Berger, and P. Colella, J. Comp. Phys. 82, 64-84 (1989).

18. C. A. Rendleman, V. E. Beckner, M. Lijewski, W. Crutchfield, and J. B. Bell, Comput. Visual Sci. 3, $147-157(2000)$.

19. R. M. More, K. H. Warren, D. A. Young, and G. B. Zimmerman, Phys. Fhids 31, 3059 (1988).

20. E. Minguez, and R. Falquina, Laser and Particle Beams 10, 651 (1992).

21. C. for Computational Science, and Engineering (2009).

22. E. Oliva, P. Zeitoun, M. Marti, P. Velarde, M. Fajardo, and K. Cassou, Journal of Physics: Conference Series 112, 042066 (4pp) (2008), URL http ://stacks . iop.org/1742-6596/112/ 042066 . 